\title{
Self-entanglement of a tumbled circular chain
}

\author{
Beatrice W. Soh $\odot,{ }^{1}$ Isabella R. Gengaro, ${ }^{1}$ Alexander R. Klotz $\odot,{ }^{1,2}$ and Patrick S. Doyle $\odot^{1, *}$ \\ ${ }^{1}$ Department of Chemical Engineering, Massachusetts Institute of Technology, Cambridge, Massachusetts 02139, USA \\ ${ }^{2}$ Department of Physics and Astronomy, California State University, Long Beach, California 90840, USA
}

(Received 30 September 2019; published 23 December 2019)

\begin{abstract}
The spontaneous knotting of linear chains has been well studied, but little attention has been given to the self-entanglement of chains with more complex topologies. In this work, we perform experiments with granular chains that undergo tumbling motion to investigate the self-entanglement of circular chains, which lack the chain ends essential for forming knots. We study the entanglement probability and types of self-entanglements formed on linear and circular chains, using the well-studied self-entanglements on a linear chain to frame our understanding of self-entanglements on a circular chain. We describe a characterization method that views a self-entangled circular chain as a link of two components and use it to characterize the self-entanglements on circular chains with known topological descriptors from knot theory. Our experimental results show that an increase in circular chain length leads to an increase in entanglement probability and entanglement complexity until a plateau is reached, similar to the trends observed with linear chains. By examining the formation pathway of several self-entanglements, we infer a general mechanism for the self-entanglement of circular chains.
\end{abstract}

DOI: 10.1103/PhysRevResearch.1.033194

\section{INTRODUCTION}

Entanglements are a common occurrence in our everyday lives. It has been theoretically proven that the knotting probability of a chain approaches unity as the chain length tends to infinity [1], hence it is inevitable that entanglements are present in a wide range of physically relevant systems. From hair and cords to DNA [2] and protein molecules [3,4], knots are indeed ubiquitous across length scales. This has led to entanglements being a subject of considerable interest among fields in science and engineering.

Although knots are mathematically well defined only in circular chains [5], linear chains with free ends can contain localized, unambiguous knots. Unlike knots on closed chains, knots on open chains can become untangled when a free end loops through the knot. Similarly, the ends of an open, unknotted chain can pass through loops on the same chain to form a knot. The spontaneous knotting and unknotting of linear polymer molecules at equilibrium has been reported in a computational study by Tubiana et al. [6]. From an experimental standpoint, single-molecule experiments have demonstrated the introduction of knots on polymers using nonequilibrium methods, including optical tweezers [7,8], electric fields [9], and compression in nanoconfinement [10]. Tying knots with optical tweezers results in knots with known topology [7,8], but this approach is low throughput and challenging. Hence, experimental studies on knotted polymers typically employ electric fields [11,12] and compression in nanoconfinement

\footnotetext{
*pdoyle@mit.edu

Published by the American Physical Society under the terms of the Creative Commons Attribution 4.0 International license. Further distribution of this work must maintain attribution to the author(s) and the published article's title, journal citation, and DOI.
}

$[10,13]$ to induce self-entanglements. Such methods, however, cannot generate knots of known topology and the entanglements formed are not resolvable by conventional fluorescence microscopy.

Given the challenge of investigating entanglements on the molecular scale, researchers have studied macroscopic model systems, which allow easier control and direct visualization, to gain insight into the physics of entanglements. Raymer and Smith [14] studied the formation of knots on string tumbled inside a rotating box and proposed a model based on random braid moves of the free ends to describe the observed distribution of knot types. Studies with entangled granular chains have shown that knots can form on freely hanging chains shaken vertically at a constant frequency [15], considered the knotting and unknotting processes on chains placed on horizontal vibrating plates $[16,17]$, examined the swelling and motion of knots on chains under tension [18], and investigated the oscillatory periodic motion of knots sedimenting in a viscous fluid [19]. Macroscale experiments provide an avenue for exploring the mechanisms of entanglements and can inform our understanding of entanglements on the microscale. From a broader perspective, granular chains can be viewed as a macroscale polymer system [20,21] and further our understanding of fundamental polymer concepts, for instance, the glass transition [22,23].

Most experimental studies on entangled polymers to date have focused on linear chains, although there is growing interest in more complex topologies, such as circular chains [24-26]. Recently, our group performed single-molecule experiments to compress and induce self-entanglements in circular DNA with electric fields, with self-entanglements serving as a minimal system for studying chain entanglements [27]. Since the conformations of the compressed circular molecules were not resolvable by fluorescence microscopy, we probed the nature of the compressed states by studying 


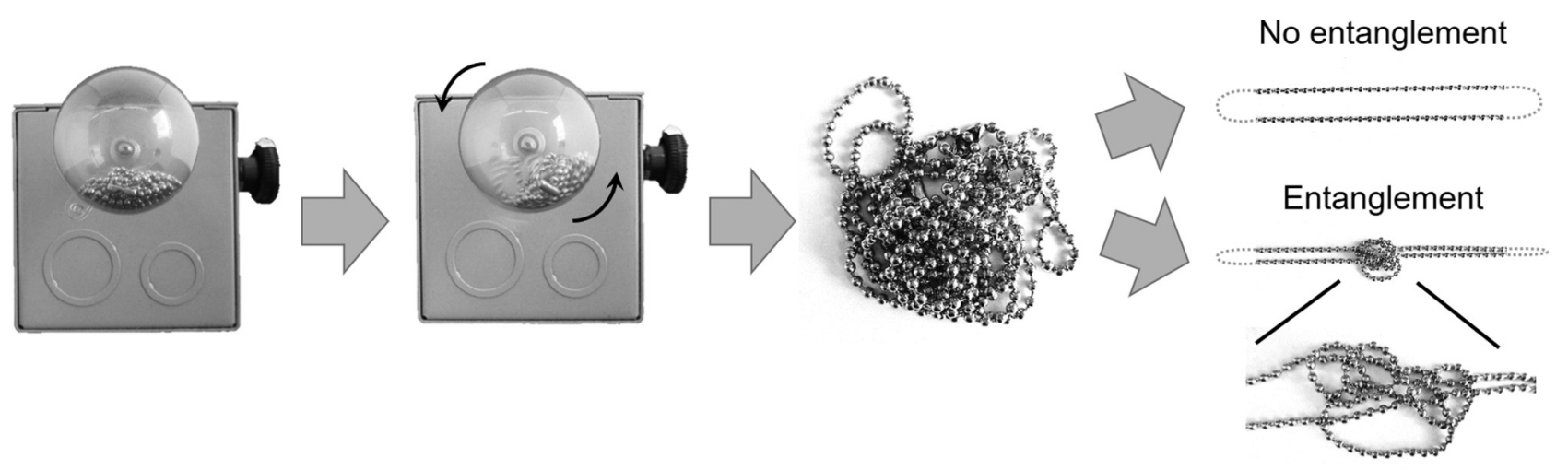

FIG. 1. Schematic for experimental procedure. The chain was placed into a plastic spherical ball mounted onto a motor shaft. The ball was rotated by a gear motor at a speed controlled by a potentiometer and for a set amount of time. The chain was then carefully transferred onto a flat surface. Using tweezers, we held either the chain ends (linear) or roughly opposite sides of the chain (circular) and pulled in opposite directions to extend the chain and evaluate for self-entanglements.

the expansion dynamics of the molecules back to equilibrium. We found that circular molecules are able to self-entangle, and strongly entangled circular molecules can remain kinetically arrested in an entangled state for time scales much longer than the polymer relaxation time. It might seem counterintuitive that circular molecules are able to self-entangle; while linear molecules can self-entangle by weaving chain ends through loops along the same chain to form intramolecular knots, circular molecules do not have chain ends.

To further investigate the physics of entanglements on circular chains, we implement a macroscopic system that enables us to directly see chain conformations. In this work, we perform experiments with granular chains that undergo tumbling motion to study self-entangled circular chains. We investigate the entanglement probability and types of self-entanglements formed on linear and circular chains, with entanglements on the linear chain providing a framework for understanding entanglements on its circular counterpart. By treating a self-entangled circular chain as a link of two components, we can use topological descriptors from knot theory-the Alexander-Briggs knot notation, Dowker-Thistlethwaite code, and linking number [28]— to characterize self-entanglements on circular chains.

\section{EXPERIMENTAL METHOD}

The experimental system consists of a two-part plastic spherical ball (diameter $=50 \mathrm{~mm}$ ) mounted onto the shaft of a gear motor, the speed of which is controlled by a potentiometer. Each chain is a series of nickel-plated metal beads (diameter $=2.4 \mathrm{~mm}$, mass $=32 \mathrm{mg}$ ) loosely connected by metal rods and a clasp at the end that allows the chain to be closed (DREAMZE, \#4336832517). The length of the chain is adjusted by connecting together different numbers of repeat units (mers), with one mer containing 185 beads and measuring $61 \mathrm{~cm}$ in length when fully extended. The flexibility of the chain can be gauged by the minimum chain length needed to form a closed loop $\xi=2 \pi / \theta_{\max }=7$, where $\theta_{\max }$ is the maximum bond angle [22,23].

The experimental procedure is summarized in Fig. 1. A granular chain was placed into the spherical ball and the ball was rotated for $10 \mathrm{~s}$ at a speed of 3.6 rotations per second about the horizontal axis perpendicular to the direction of gravity, causing the chain to undergo a tumbling motion [see Supplemental Material (SM) for Movies S1-S3 [29]). The rotation of the ball was started and stopped manually with an on/off switch. After rotation was stopped, the chain was carefully removed from the ball and placed onto a flat surface. We then picked out either the chain ends (linear) or roughly opposite sides of the chain (circular) with tweezers and pulled the chain horizontally in opposite directions to extend the chain and enable direct observation of any entanglements, similar to protocols used in computational [30] and experimental studies of knot formation $[9,10]$. If the chain contained self-entanglements, we gently spread the chain apart near the entanglement to allow all chain crossings to be seen. The entanglement was then digitally photographed. Both linear and circular chains were studied, and the length of the chain was varied from 1 to 3.5 mers. For each set of parameters, we conducted 60 trials and calculated the resulting probability of entanglement.

From a mathematical standpoint, the topological state of a linear chain is not rigorously defined, as the free ends of the chain can thread through loops and change the chain topology. The ends of the chain have to be joined to form a closed loop for topological classification, and this was facilitated by extending the free ends of a linear chain outward from the center of mass of the chain in the experiments. On the other hand, the topological state of a circular chain cannot be changed without opening the chain, accordingly the circular chains in our experiments remained unknotted throughout the tumbling process. Nevertheless, we observed self-entanglements on the tumbled circular chains that, akin to knots on linear chains, would tighten upon pulling on opposite sides of the chain. Such self-entanglements on circular chains include known entanglements, such as slipknots. See SM [29] for a discussion on ensuring the experimental protocol had minimal influence on the measured entanglement probability.

\section{RESULTS AND DISCUSSION}

\section{A. Entanglement probability}

Figure 2 shows the probability of entanglement as a function of chain length for linear and circular chains. For both 


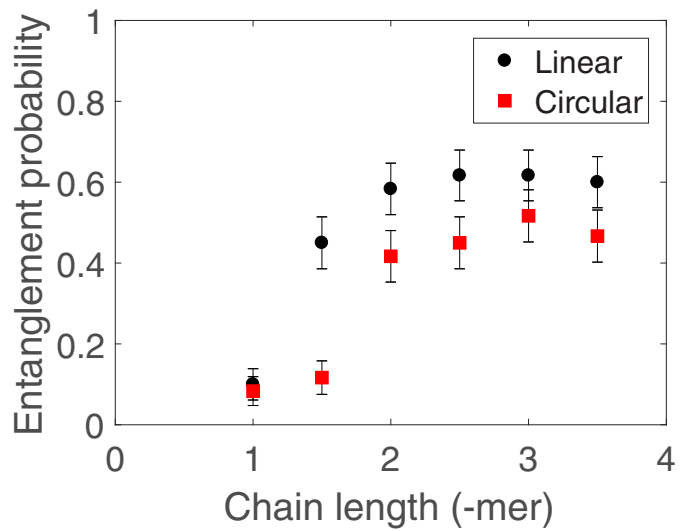

FIG. 2. Probability of entanglement as a function of chain length for linear and circular chains rotated at 3.6 rotations per second for $10 \mathrm{~s}$. Each data point represents the probability of chain entanglement over 60 trials. Error bars represent standard error.

chain topologies, the probability of entanglement increases sharply with an increase in chain length until it reaches a plateau. This result is consistent with computational studies that demonstrated the probability of not forming a knot for self-avoiding walks decreases exponentially with contour length [31,32]. The observed probability of entanglement, however, does not approach unity as has been proven mathematically [1]. This phenomenon was similarly observed in previous experimental investigations into the knotting of macroscale linear chains [14,17]. Hickford et al. [17] found that the knotting time for granular chains is independent of chain length, while the unknotting time increases significantly with chain length. The balance of the knotting and unknotting processes then gives rise to the knotting probability within a finite time. Raymer and Smith [14] varied several parameters in experiments with tumbling string and observed that the knotting probability saturated at different values depending on agitation time and string flexibility, which suggests that the knotting probability tends to $100 \%$ with long string length, prolonged agitation time, and high string flexibility. While we did not vary chain flexibility, we note that increasing tumbling time led to a substantial increase in entanglement probability for both linear and circular chains (see Table S1 in SM [29]), suggesting that the formation of entanglements is kinetically limited in our experiments.

Given that the extension of a circular chain is half that of its linear counterpart, one might expect the curves of entanglement probability for linear and circular chains to overlap when plotted against effective contour length (see SM for rescaled curves). However, with the entanglement probabilities reaching different saturation values at long chain lengths ( $\approx 60 \%$ for linear versus $\approx 50 \%$ for circular), it is worth noting that the entanglement probabilities for linear and circular chains do not collapse onto a master curve.

\section{B. Classification of knots on linear chains}

One of the central questions in knot theory is the classification of knots. To determine whether two knots are equivalent, knot invariants can be calculated from the corresponding knot diagrams, i.e., projections of the knot onto a plane. Commonly used knot invariants are the Alexander, Jones, and HOMFLY polynomials [28]. An alternative to computing knot invariants is to obtain the algebraic Dowker-Thistlethwaite code of the knot and determine the knot type from lookup tables [33].

The advantage of implementing macroscale systems is the ability to directly visualize the chain conformation and identify the types of self-entanglements formed [14,15]. To determine the types of knots formed on linear chains in this study, we calculate the Dowker-Thistlethwaite code for the knot and compare it against a lookup table of prime knots with up to 15 crossings [34]. For each knot, we connect the chain ends and draw a knot diagram with an arbitrary orientation. We traverse the knot diagram from a random starting point and label each crossing point in ascending order, adding a negative sign to an even number if the corresponding strand is an over-crossing. Each crossing is encountered twice and hence labeled by a pair of integers, one odd and one even. The Dowker-Thistlethwaite code for the knot is obtained as the sequence of even integers associated with the odd integers in ascending order [33].

Figure 3 shows several examples of knots on linear chains and identification of the knot types via calculation of the Dowker-Thistlethwaite code. We identify $\approx 92 \%$ of the knots formed on linear chains in this study (163 out of 178) as prime knots with minimal crossing numbers between 3 and 15. At least half of the unclassified knots can be visually identified to be composite knots (e.g., $3_{1} \# 3_{1}$ and $3_{1} \# 4_{1}$ ) and the remaining knots contain a substantial number of crossings, which makes visualization difficult (see Fig. S3 in SM). We highlight that a large majority of the knots formed are prime knots, a finding consistent with the results of the study by Raymer and Smith, in which $\approx 96 \%$ of all knots were classified to be prime knots [14]. Among the prime knots formed, the most commonly observed knot was the $33_{1}$ knot, followed by the $4_{1}$ and $5_{2}$ knots. Between the two knots with a minimal crossing number of 5 , the $5_{2}$ knot was observed much more frequently than the $5_{1}$ knot $(\approx 12 \%$ vs $\approx 3 \%$ of all knots). Interestingly, several computational studies investigating the probabilities of knot types in different random-walk models have reported the probability of the $5_{2}$ knot to be higher than that of the $5_{1}$ knot [35-38]. We conjecture that our experimental setup might lead to the favored formation of twist knots over torus knots; the flexibility of the chain coupled with the rotating motion of the ball encourages the chain to continuously twist itself as it tumbles, which facilitates the formation of twist knots. It should be noted that there is no apparent bias favoring the formation of chiral versus achiral knots, or left versus right enantiomers of a chiral knot (see SM [29]).

\section{Topological characterization of self-entanglements on circular chains}

While knots have been well studied and can be classified using knot invariants, self-entanglements on closed, unknotted chains have received little attention. In this work, we seek to characterize self-entanglements on unknotted circular chains, i.e., circular entanglements, using known topological descriptors from knot theory. Figure 4 depicts the characterization method used for self-entanglements on circular chains in this 
(a)

$3_{1}$
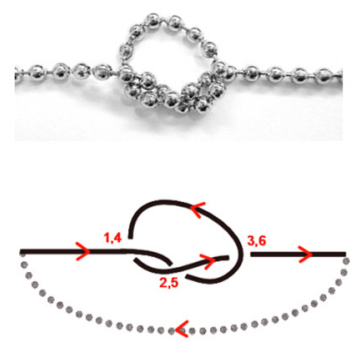

$[4,6,2]$ (b)
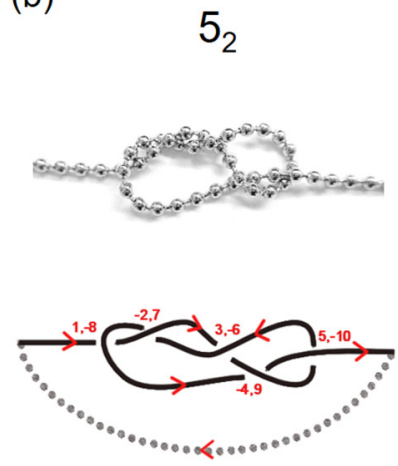

$[-8,-6,-10,-2,-4]$ (c)

$8_{4}$
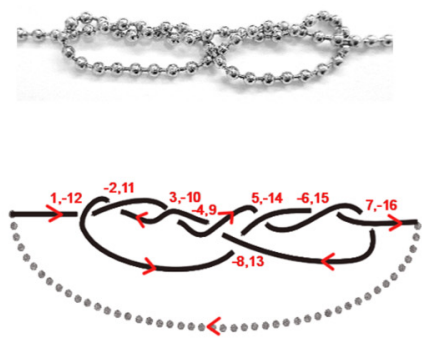

$[-12,-10,-14,-16,-4,-2,-8,-6]$ (d)

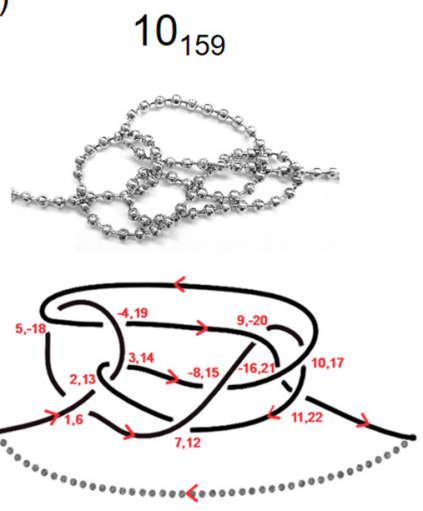

FIG. 3. Identification of knot types (a) $3_{1}$, (b) $5_{2}$, (c) $8_{4}$, and (d) $10_{159}$ on linear chains using algebraic descriptors of knots. Each knot was digitally photographed (top) and the Dowker-Thistlethwaite code can be determined from the knot diagram (bottom).

work. The characterization method is broadly inspired by the analysis of protein structures for the presence of slipknots, which recognizes that the configuration of a slipknot contains knotted subchains although the whole chain is unknotted $[39,40]$. Similarly, a self-entangled circular chain is unknotted as a whole, but parts of the chain can contain knotted structures. A self-entanglement on a stretched circular chain (Fig. 1) can be viewed as a tangle with two strands and four ends [41]. To obtain a proper topological link, we close each curve with an auxiliary arc, analogous to the procedure used to convert a pair of open chains into a physical link [42]. Starting from any end, we can traverse a strand until another end is reached and connect these two ends to form a closed loop. The same process can be repeated with the other strand and its two ends. With this, a circular entanglement can be viewed as a link of two components and we can then determine the knot type on each component, as well as the linking number of the link. We note that links constructed from the circular entanglements are more complex than the topologically classified two-component links described by the Alexander-Briggs notation.

We demonstrate how self-entanglements on circular chains can be characterized using as an example the most commonly observed self-entanglement, shown in Fig. 4(a), which one might recognize as a slipknot. We start with the top right end and walk along the black strand in a clockwise direction until we arrive at the top left end. Joining the two ends to form a closed loop, we identify the black curve to be a $3_{1}$ knot. The identification of more complex knot types can be achieved via calculation of the Dowker-Thistlethwaite code. Next, we start at the bottom left end and traverse the opposite red strand in a clockwise direction to reach the bottom right end. Closing the ends into a loop, we identify the red curve to be a $0_{1}$ knot, or an unknot. We can then use a link invariant-the linking number $\mathrm{L}_{\mathrm{k}}$ - to describe how the two components are linked. The linking number is an approximate measure of how many times one component winds around another and is computed as half the sum of signs of crossings between the two components [43]. We consider the absolute value of the linking number, which is independent of the choice of orientations of the two curves. The linking number of the black and red components in Fig. 4(a) is determined to be 1 . We label this self-entanglement as $\left(3_{1}, 0_{1}, 1\right)$, where the first two terms are the knot types of the two curves as denoted by the Alexander-Briggs notation and the third term is the linking number of the components. (a)

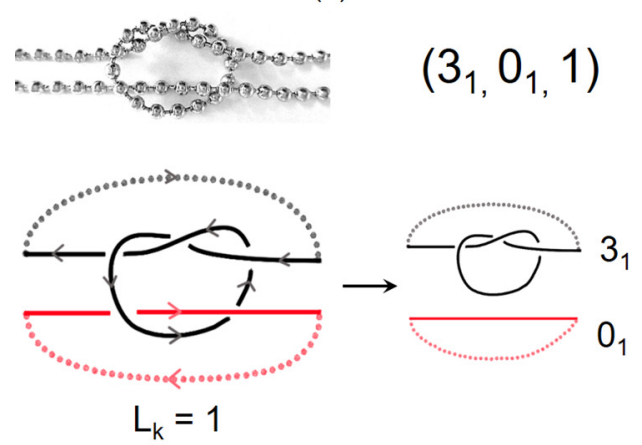

(b)

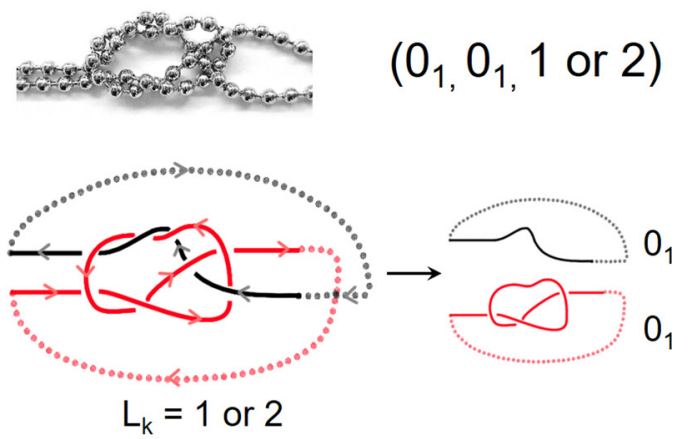

FIG. 4. Characterization method for circular entanglements demonstrated with (a) $\left(3_{1}, 0_{1}, 1\right)$ and (b) $\left(0_{1}, 0_{1}, 1\right.$ or 2$)$. A circular entanglement (top) is viewed as a link of two components (bottom), which allows the determination of the knot type on each component and the linking number of the link. Since the components in the link are closed with auxiliary arcs and not strictly closed curves, the linking number can take on two possible values in certain cases. 


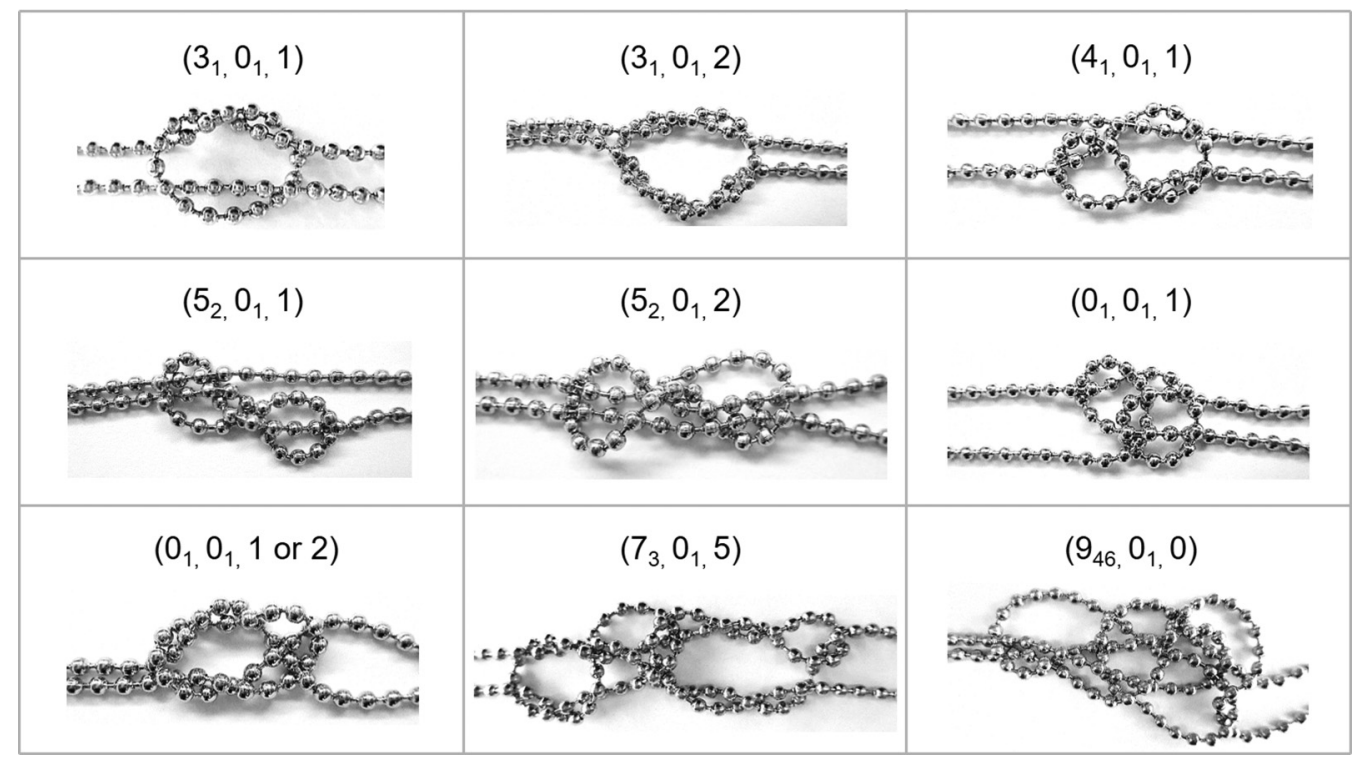

FIG. 5. Examples of circular entanglements characterized by the characterization method.

Since there must be an even number of crossings between two components of a link, the linking number is always an integer. In the case of self-entanglements on circular chains, however, because the opposing strands are not strictly closed curves and instead closed with auxiliary arcs, one component does not necessarily cross the other an even number of times. Figure 4(b) shows an example of a circular entanglement that cannot be characterized by a single linking number. Following the characterization protocol, we identify the black and red curves to be $0_{1}$ knots. The two components cross each other five times, which results in a linking number of either 1 or 2 , depending on the sign of the nonexistent crossing.

Using the characterization method, we are able to characterize $96 \%$ of the self-entanglements formed on circular chains in this study (118 out of 123). The rest of the circular entanglements contain a large number of crossings, which makes visualization difficult (see Fig. S4 in SM). Among the characterized self-entanglements, the identified knot types on individual curves have minimal crossing numbers ranging from 0 to 12 and the links have linking numbers between 0 and 5. Note that a linking number of 0 does not necessarily mean the components are unlinked; the Whitehead link is an example of linked curves that have a linking number of 0 . The most commonly observed circular entanglement was $\left(3_{1}, 0_{1}, 1\right)$, followed by $\left(4_{1}, 0_{1}, 1\right)$, accounting for $37 \%$ and 9\% of the circular entanglements respectively. The frequent occurrence of $\left(3_{1}, 0_{1}, 1\right)$ suggests that it is the simplest selfentanglement that can be formed on circular chains. We point out that $4 \%$ of the circular entanglements consist of multiple entanglements, such as a pair of $\left(3_{1}, 0_{1}, 1\right)$ on the same chain, that can be seen as being analogous to composite knots on a linear chain. Figure 5 shows various self-entanglements on circular chains and how they are characterized by our characterization method. Through the utilization of simple topological descriptors from knot theory, we can characterize complex self-entanglements with many crossings, for example $\left(7_{3}, 0_{1}, 5\right)$ and $\left(9_{46}, 0_{1}, 0\right)$. Furthermore, we find that it is possible to distinguish between circular entanglements that appear to be similar, such as $\left(3_{1}, 0_{1}, 1\right)$ and $\left(3_{1}, 0_{1}, 2\right)$, as well as $\left(5_{2}, 0_{1}, 1\right)$ and $\left(5_{2}, 0_{1}, 2\right)$.

Since the characterization method is based on envisioning the self-entanglement as a tangle with two opposing strands, we imagine that the way in which the circular chain was extended to allow for visualization of the entanglement can affect the precise characterization of a complex entanglement. We highlight that the goal of the characterization method is not to fully classify self-entanglements on circular chains, but to be used as a way to systematically gauge the complexity of a circular entanglement. Indeed, the characterization method is unable to uniquely classify different types of circular entanglements. Figure 6 shows an example of two noticeably different circular entanglements that have the same characterization. Both entanglements contain $3_{1}$ and $0_{1}$ knots on the individual curves and the linking number of the link is 1 , yet one readily observes the distinction between the entanglements. It is possible to employ more topological descriptors to achieve a more fine-grained characterization of the entanglements. For example, we can exploit the concept of writhe from the mathematical description of supercoiled DNA [44] to distinguish between the two entanglements shown in Fig. 6. However, for the purpose of this study, the characterization method at present suffices to broadly categorize the circular entanglements observed and give a rough measure of the complexity of circular entanglements generated in the experiments. While the method is not a mathematically rigorous classification scheme, it can form a conceptual foundation for thinking about self-entanglements on circular chains and to be further built upon.

\section{Types of self-entanglements}

Having systematically characterized the selfentanglements formed on linear and circular chains, we now consider the types and complexity of self-entanglements formed as the chain length is varied. For this analysis, we ignore composite and unidentifiable entanglements. 

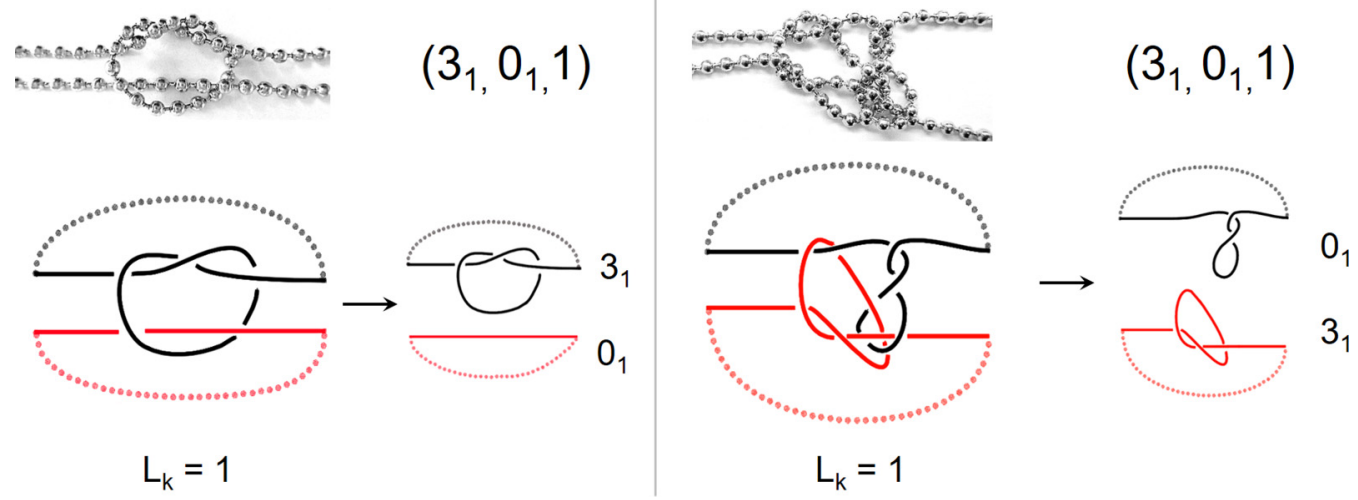

FIG. 6. Two distinct circular entanglements that have the same characterization.

Figure 7(a) shows the number of different types of entanglements observed on linear and circular chains as a function of chain length, normalized by the total number of unique entanglements observed across all experiments for the

(a)

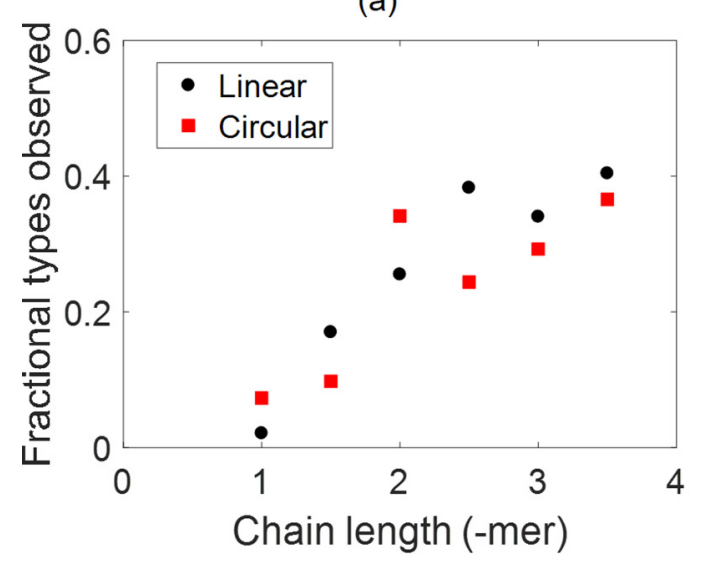

(b)

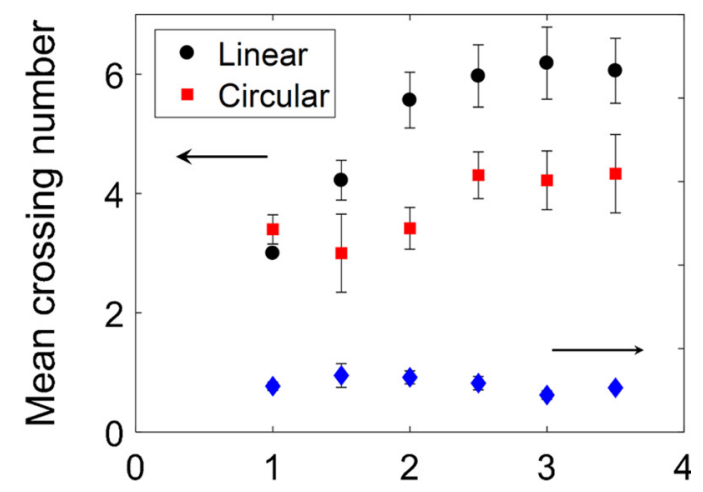

FIG. 7. (a) Fractional number of unique entanglements observed as a function of chain length for linear and circular chains. (b) Left axis: Mean minimal crossing number as a function of chain length for linear and circular chains. Right axis: Mean linking number as a function of chain length for circular chains (blue diamond). The linking numbers of circular entanglements with ambiguous linking numbers are reported as the average of the possible linking numbers. Error bars represent standard error. corresponding chain topology. For both linear and circular chains, we observe an increase in the number of unique entanglements with chain length until a plateau is reached, similar to the saturation of knotting probability at long chain lengths.

A standard measure of knot complexity is the minimal crossing number of the knot. To gauge the complexity of a circular entanglement, we sum up the minimal crossing numbers of the knots identified on opposing strands of the entanglement. For example, we consider the minimal crossing number of $\left(3_{1}, 0_{1}, 1\right)$ to be 3 . In Fig. $7($ b), we plot the mean minimal crossing number as a function of chain length for linear and circular chains. The mean minimal crossing number for both linear and circular chains rises sharply with increasing chain length and plateaus at long chain lengths, akin to the number of unique entanglements observed and entanglement probability. Like knots formed on linear chains, self-entanglements formed on circular chains become more complex as the chain length becomes longer. While the mean minimal crossing number exhibits a dependence on chain length, the mean linking number for circular chains [Fig. 7(b)] does not vary significantly across chain length. This is perhaps unsurprising, as the linking number is a simple invariant that gives only a rough measure of how two components are linked together. It is easy to see that two curves can wind about each other in both directions and negate the sum of crossing signs, which is why a linking number of 0 does not indicate the components are disjointed. Hence, we highlight that the linking number is a useful tool for characterizing circular entanglements, but it does not necessarily reflect complexity of the entanglement. See SM [29] for histograms of minimal crossing number and linking number for self-entanglements on linear and circular chains of various chain lengths.

\section{E. Mechanism for self-entanglement of circular chains}

The ability of circular chains to self-entangle in the absence of chain ends motivates us to conjecture the mechanism of forming self-entanglements on circular chains. To gain insight into the possible entanglement mechanisms, we consider in detail pathways to form several simple self-entanglements on circular chains (Fig. 8). As shown in Figs. 8(a) and 8(b), the formation of the simplest and most commonly observed 
(a)
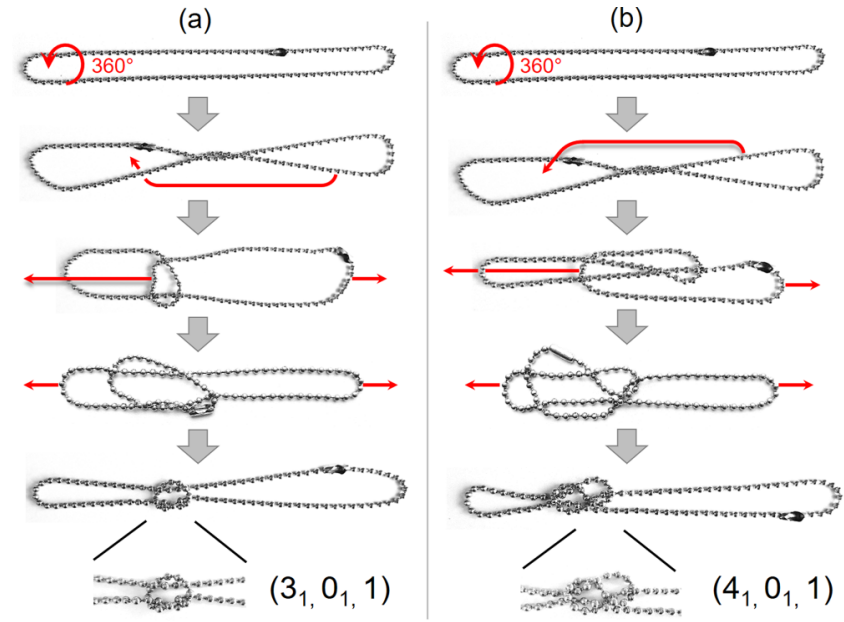

(c)
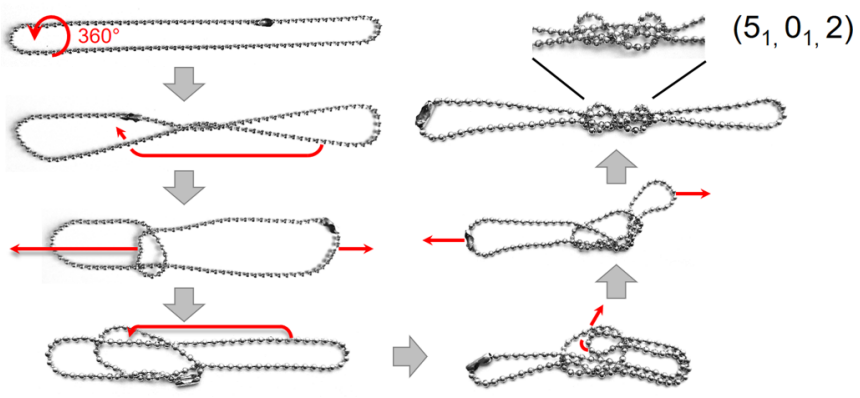

FIG. 8. Examples of possible pathways for forming selfentanglements on circular chains. (a) $\left(3_{1}, 0_{1}, 1\right)$; (b) $\left(4_{1}, 0_{1}, 1\right)$; (c) $\left(5_{1}, 0_{1}, 2\right)$.

self-entanglements- $\left(3_{1}, 0_{1}, 1\right)$ and $\left(4_{1}, 0_{1}, 1\right)$-involves twisting of the chain and passing of a double-folded segment through another double-folded segment on the same chain. The configuration in which a double-folded segment opens up and penetrates another double-folded segment on the same chain is known as self-threading, and threadings have been reported to slow down ring polymer dynamics both computationally [45-47] and experimentally [27]. It is interesting to observe the disparity in occurrences of $\left(3_{1}, 0_{1}, 1\right)$ and $\left(4_{1}, 0_{1}, 1\right)$ despite the degree of similarity in entanglement pathways, with the only difference being a loop threaded under or over through another loop. In an investigation into knot formation on freely hanging chains shaken vertically, Belmonte et al. [15] found that the $4_{1}$ knot occurs more frequently than the $3_{1}$ knot, a result of the $3_{1}$ knot untying more easily. We recognize that the probability of a given entanglement occurring depends on not only the entanglement pathway, but also the untangling dynamics.

The least complex circular entanglements result from a combination of twisting and threading of the chain, which suggests that the formation of more complex selfentanglements is built upon a series of twists and threadings. We delve into the formation pathway of a more complex selfentanglement $\left(5_{1}, 0_{1}, 2\right)$, depicted in Fig. 8(c). The first half of the pathway is the same as that for $\left(3_{1}, 0_{1}, 1\right)$, and the second half involves a double-folded segment weaving through another double-folded segment within the growing tangle. With the addition of one self-threading, the circular entanglement becomes moderately complex compared to $\left(3_{1}, 0_{1}, 1\right)$. We can imagine that a combination of just a few chain twists and threadings is sufficient to build up to a complex circular entanglement. While the entanglement pathways illustrated in Fig. 8 may not represent the only ways in which the given self-entanglements can form on circular chains, the possible mechanisms shed light on what gives rise to circular entanglements. In our recent experimental study on self-entangled circular DNA [27], we postulated that the self-entangled states were induced by self-threadings, but were unable to confirm this by direct visualization of individual molecules given the length scales involved. The possibility that circular molecules can self-entangle by forming double-folded knots was deemed negligible due to the energetically unfavorable configuration of having two halves of the chain align together [48]. Our hypothesis is supported by the results of the current study, which suggests that circular entanglements originate from a series of chain twists and self-threadings.

\section{CONCLUSION}

In this work, we studied the self-entanglement of circular chains using a macroscale system of granular chains tumbled in a rotating ball. We investigated the probability of forming self-entanglements on circular chains and found that the entanglement probability increases sharply with chain length until it reaches a plateau at long chain length, much like the entanglement probability on linear chains. Using a characterization method based on treating a self-entangled circular chain as a link of two components, we were able to characterize the majority of circular entanglements formed with known topological descriptors from knot theory. We used the sum of minimal crossing numbers of knots on the two curves comprising a circular entanglement as a measure of entanglement complexity and observed an increase in entanglement complexity with an increase in chain length for circular chains, similar to the trend seen with linear chains. By hypothesizing how the simplest self-entanglements form on circular chains, we acquired an understanding of the mechanism for forming circular entanglements. Self-entanglements on circular chains generally arise from a series of twisting and threading of the chain, with just a few twists and threadings sufficient to induce a complex self-entanglement.

The results of this study highlight the advantages of implementing macroscale systems to probe microscale systems. Previous single-molecule DNA experiments performed by our group [27] concluded that circular molecules can selfentangle and remain in an entangled state for long time scales, but due to the resolution limit of the optical system, the nature of the self-entangled states could only be inferred from relaxation dynamics. With a macroscale system of granular chains, we are able to directly visualize the chain conformation and examine the self-entanglements on circular chains. Furthermore, it is much easier to control and systematically vary experimental parameters with macroscale systems in comparison to microscale experiments. Indeed, macroscale systems have gained recent interest as tools to explore a wide range of topics in polymer physics [18,49]. Looking 
forward, we hope that this work motivates further studies into the entanglements of macroscale chains, for example, the untangling dynamics of self-entanglements on circular chains or self-entanglements on chains with other complex topologies.

[1] D. W. Sumners and S. G. Whittington, Knots in self-avoiding walks, J. Phys. A: Math. Gen 21, 1689 (1988).

[2] J. Arsuaga, M. Vázquez, S. Trigueros, D. W. Sumners, and J. Roca, Knotting probability of DNA molecules confined in restricted volumes: DNA knotting in phage capsids, Proc. Natl. Acad. Sci. USA 99, 5373 (2002).

[3] M. L. Mansfield, Are there knots in proteins? Nat. Struct. Biol. 1, 213 (1994).

[4] W. R. Taylor, A deeply knotted protein structure and how it might fold, Nature 406, 916 (2000).

[5] E. Orlandini and S. G. Whittington, Statistical topology of closed curves: Some applications in polymer physics, Rev. Mod. Phys. 79, 611 (2007).

[6] L. Tubiana, A. Rosa, F. Fragiacomo, and C. Micheletti, Spontaneous knotting and unknotting of flexible linear polymers: Equilibrium and kinetic aspects, Macromolecules 46, 3669 (2013).

[7] Y. Arai, R. Yasuda, Ken-ichirou Akashi, Y. Harada, H. Miyata, K. Kinosita, and H. Itoh, Tying a molecular knot with optical tweezers, Nature 399, 446 (1999).

[8] X. R. Bao, H. J. Lee, and S. R. Quake, Behavior of Complex Knots in Single DNA Molecules, Phys. Rev. Lett. 91, 265506 (2003).

[9] J. Tang, N. Du, and P. S. Doyle, Compression and selfentanglement of single DNA molecules under uniform electric field, Proc. Natl. Acad. Sci. USA 108, 16153 (2011).

[10] S. Amin, A. Khorshid, L. Zeng, P. Zimny, and W. Reisner, A nanofluidic knot factory based on compression of single DNA in nanochannels, Nat. Commun. 9, 1506 (2018).

[11] B. W. Soh, V. Narsimhan, A. R. Klotz, and P. S. Doyle, Knots modify the coil-stretch transition in linear DNA polymers, Soft Matter 14, 1689 (2018).

[12] A. R. Klotz, B. W. Soh, and P. S. Doyle, Motion of Knots in DNA Stretched by Elongational Fields, Phys. Rev. Lett. 120, 188003 (2018).

[13] R. Metzler, W. Reisner, R. Riehn, R. Austin, J. O. Tegenfeldt, and I. M. Sokolov, Diffusion mechanisms of localised knots along a polymer, Europhys. Lett. 76, 696 (2006).

[14] D. M. Raymer and D. E. Smith, Spontaneous knotting of an agitated string, Proc. Natl. Acad. Sci. USA 104, 16432 (2007).

[15] A. Belmonte, M. J. Shelley, S. T. Eldakar, and C. H. Wiggins, Dynamic Patterns and Self-Knotting of a Driven Hanging Chain, Phys. Rev. Lett. 87, 114301 (2001).

[16] E. Ben-Naim, Z. A. Daya, P. Vorobieff, and R. E. Ecke, Knots and Random Walks in Vibrated Granular Chains, Phys. Rev. Lett. 86, 1414 (2001).

[17] J. Hickford, R. Jones, S. Courrech du Pont, and J. Eggers, Knotting probability of a shaken ball-chain, Phys. Rev. E 74, 052101 (2006).

[18] Y. C. Chou, Observations of metastable states of the free swelling knots and directional motion of tensioned knots in vibrated bead chains, Eur. Phys. J. E 42, 79 (2019).

\section{ACKNOWLEDGMENTS}

We thank Liang Dai for useful discussions. This work was supported by the National Science Foundation (NSF) Grant No. CBET-1936696. B.W.S. is funded by the Agency for Science, Technology and Research (A*STAR), Singapore.

[19] M. Gruziel, K. Thyagarajan, G. Dietler, A. Stasiak, M. L. Ekiel-Jeżewska, and P. Szymczak, Periodic Motion of Sedimenting Flexible Knots, Phys. Rev. Lett. 121, 127801 (2018).

[20] K. Safford, Y. Kantor, M. Kardar, and A. Kudrolli, Structure and dynamics of vibrated granular chains: Comparison to equilibrium polymers, Phys. Rev. E 79, 061304 (2009).

[21] S. Tricard, E. Feinstein, R. F. Shepherd, M. Reches, P. W. Snyder, D. C. Bandarage, M. Prentiss, and G. M. Whitesides, Analog modeling of Worm-Like Chain molecules using macroscopic beads-on-a-string, Phys. Chem. Chem. Phys. 14, 9041 (2012).

[22] L.-N. Zou, X. Cheng, M. L. Rivers, H. M. Jaeger, and S. R. Nagel, The packing of granular polymer chains, Science 326, 408 (2009).

[23] P.-P. Wen, N. Zheng, L.-S. Li, H. Li, G. Sun, and Q.-F. Shi, Polymerlike statistical characterization of two-dimensional granular chains, Phys. Rev. E 85, 031301 (2012).

[24] R. M. Robertson and D. E. Smith, Strong effects of molecular topology on diffusion of entangled DNA molecules, Proc. Natl. Acad. Sci. USA 104, 4824 (2007).

[25] M. Abadi, M. F. Serag, and S. Habuchi, Single-molecule imaging reveals topology dependent mutual relaxation of polymer chains, Macromolecules 48, 6263 (2015).

[26] Y. Zhou, K.-W. Hsiao, K. E. Regan, D. Kong, G. B. McKenna, R. M. Robertson-Anderson, and C. M. Schroeder, Effect of molecular architecture on ring polymer dynamics in semidilute linear polymer solutions, Nat. Commun. 10, 1753 (2019).

[27] B. W. Soh, A. R. Klotz, R. M. Robertson-Anderson, and P. S. Doyle, Long-Lived Self-Entanglements in Ring Polymers, Phys. Rev. Lett. 123, 048002 (2019).

[28] K. E. Horner, M. A. Miller, J. W. Steed, and P. M. Sutcliffe, Knot theory in modern chemistry, Chem. Soc. Rev. 45, 6432 (2016).

[29] See Supplemental Material at http://link.aps.org/supplemental/ 10.1103/PhysRevResearch.1.033194 for representative movies, effects of experimental protocol on entanglement probability, discussion on entanglement chirality and additional data analysis.

[30] L. Dai and P. S. Doyle, Universal knot spectra for confined polymers, Macromolecules 51, 6327 (2018).

[31] J. P. J. Michels and F. W. Wiegel, On the topology of a polymer ring, Proc. R. Soc. A 403, 269 (1986).

[32] K. Koniaris and M. Muthukumar, Self-entanglement in ring polymers, J. Chem. Phys. 95, 2873 (1991).

[33] C. H. Dowker and M. B. Thistlethwaite, Classification of knot projections, Topol. Appl. 16, 19 (1983).

[34] Knot ID, http://inclem.net/knotidentifier/.

[35] T. Deguchi and K. Tsurusaki, A statistical study of random knotting using the vassiliev invariants, J. Knot Theory Ramif. 03, 321 (1994). 
[36] V. Katritch, W. K. Olson, A. Vologodskii, J. Dubochet, and A. Stasiak, Tightness of random knotting, Phys. Rev. E 61, 5545 (2000).

[37] J. Arsuaga, M. Vazquez, P. McGuirk, S. Trigueros, D. W. Sumners, and J. Roca, DNA knots reveal a chiral organization of DNA in phage capsids, Proc. Natl. Acad. Sci. USA 102, 9165 (2005).

[38] C. Micheletti, D. Marenduzzo, E. Orlandini, and D. W. Sumners, Simulations of knotting in confined circular DNA, Biophys. J. 95, 3591 (2008).

[39] N. P. King, E. O. Yeates, and T. O. Yeates, Identification of rare slipknots in proteins and their implications for stability and folding, J. Mol. Biol. 373, 153 (2007).

[40] J. I. Sułkowska, E. J. Rawdon, K. C. Millett, J. N. Onuchic, and A. Stasiak, Conservation of complex knotting and slipknotting patterns in proteins, Proc. Natl. Acad. Sci. USA 109, E1715 (2012).

[41] J. R. Goldman and L. H. Kauffman, Rational Tangles, Adv. Appl. Math. 18, 300 (1997).

[42] M. Caraglio, C. Micheletti, and E. Orlandini, Physical Links: defining and detecting inter-chain entanglement, Sci. Rep. 7, 1156 (2017).
[43] C. Livingston, Enhanced linking numbers, Am. Math. Mon. 110, 361 (2003).

[44] S. M. Mirkin, DNA Topology: Fundamentals, In Encyclopedia of Life Sciences, (Wiley, New York, 2001).

[45] E. Lee, S. Kim, and Y. J. Jung, Slowing down of ring polymer diffusion caused by inter-ring threading, Macromol. Rapid Commun. 36, 1115 (2015).

[46] D. G. Tsalikis, V. G. Mavrantzas, and D. Vlassopoulos, Analysis of slow modes in ring polymers: Threading of rings controls long-time relaxation, ACS Macro Lett. 5, 755 (2016).

[47] D. Michieletto and M. S. Turner, A topologically driven glass in ring polymers, Proc. Natl. Acad. Sci. USA 113, 5195 (2016).

[48] S. Gorczyca, C. D. Chapman, and R. M. Robertson-Anderson, Universal scaling of crowding-induced DNA mobility is coupled with topology-dependent molecular compaction and elongation, Soft Matter 11, 7762 (2015).

[49] F. Samadi Taheri, H. Fazli, M. Doi, and M. Habibi, Granular chain escape from a pore in a wall in the presence of particles on one side: A comparison to polymer translocation, Soft Matter 14, 5420 (2018). 